\title{
Proyectos de gobierno y desarrollo de institucionalidad juvenil: intensidades y tensiones del vínculo en Argentina (I989-20I5)
}

\author{
Eduardo Javier Pereyra, Ph.D. \\ Becario posdoctoral de Conicet, Argentina*
}

eduardopereyra_24@hotmail.com

\section{Resumen (descriptivo)}

En su carácter de revisión de tema, el objetivo central del artículo consiste en indagar sobre el vínculo existente entre un tipo de proyecto de gobierno y el desarrollo que el Estado nacional hace de institucionalidad juvenil. Centrándose en el caso argentino, se analiza si, dado el "giro a la izquierda» que representó el proyecto de gobierno kirchnerista (2003-2015), existieron rupturas significativas en la producción de institucionalidad pública juvenil en el ámbito nacional, en comparación con lo desarrollado en la materia por los gobiernos durante la «larga década neoliberal» (1989-2003). El presente trabajo estudia, a partir de fuentes secundarias, el nivel de la administración que se ocupa específicamente de los problemas de la juventud, es decir, los organismos gubernamentales de juventud. Para ello se incluye un análisis cualitativo de las lógicas con que se aborda la cuestión juvenil en los programas y dispositivos que el organismo implementa, capacidades estatales desarrolladas y el rol y peso político del organismo en el entramado institucional general de las políticas juveniles implementadas por el Estado nacional.

\section{Palabras clave}

Estado, juventud, institución política, política gubernamental.

\section{Thesauro}

Tesauro de ciencias sociales de la Unesco.

\section{Para citar este artículo}

Pereyra, E. J. (2020). Proyectos de gobierno y desarrollo de institucionalidad juvenil: intensidades y tensiones del vínculo en Argentina (1989-2015). Revista Latinoamericana de Ciencias Sociales, Niñez y Juventud, 18(1), 1-31. http://dx.doi.org/ $10.11600 / 1692715 x .18108$

\section{Historial}

Recibido: 24.05.2019

Aceptado: 23.07.2019

Publicado: 06.12.2019

\section{Información artículo}

Artículo fruto de la asistencia a paneles, talleres y debates ocurridos en la VII Escuela Internacional de la Red Iberoamericana de Posgrados en Infancia y Juventud «Migraciones, violencias, estigmas y resistencias en infancias y juventudes». A su vez, la discusión expuesta se inscribe, en parte, en un trabajo de investigación más amplio dentro de una beca doctoral financiada por Conicet (1/08/2014- 31/03/2019), denominada «Desarrollo de sistemas de protección social y bienestar juvenil en el ámbito municipal de la provincia de Córdoba», aprobada el 14 de mayo de 2014, código 2172014000957900. Área: Ciencias Políticas. Subárea: Administración Pública. 


\section{Government projects and development of youth institutions: intensities and tensions Argentina (I989-20I5)}

\section{Abstract (descriptive)}

The main objective of the article is to investigate the links between a type of government project and the development of youth institutions. Focusing on Argentina, the article analyzes whether in the «shift to the left» represented by the Kirchner governments (2003-2015) there were important shifts in the work of youth public institutions at a national level compared to what was achieved by different national governments during the «long neoliberal decade» (1989-2003). The present study is limited to analyzing secondary sources to identify the level of public administration specifically focused on youth issues, Government Youth Organizations. A qualitative analysis has been included on the logic of addressing youth issues using the programs and mechanisms employed by the agency, developing state capacities and the role and political weight of the agency in the general institutional framework of youth policies implemented by the State.

Keywords

State, Youth, Political Institution, Government Policy.

\section{Projetos do governo e desenvolvimento da institucionalidade da juventude: intensidades e tensões do vínculo na Argentina (I989-20I5)}

\section{Resumo (descritivo)}

Em sua natureza de revisão de assunto, o principal objetivo do artigo é investigar a ligação entre um tipo de projeto governamental e o desenvolvimento de instituições de juventude pelos Estados nacionais. Focalizando o caso argentino, analisa-se que diante da «curva esquerda» representada pelo projeto do governo Kirchnerista (2003-2015) ocorreram importantes interrupções na produção da institucionalidade pública juvenil em nível nacional, em relação ao que foi implantado pelos governos durante a «longa década neoliberal» (1989-2003). O presente trabalho limita-se à abordagem, a partir de fontes secundárias, do nível da administração especificamente ocupada com problemas da juventude, isto é, às Organizações Juvenis do Governo. Para este propósito, uma análise qualitativa é realizada sobre a lógica de abordar a questão da juventude através dos programas e dispositivos que o organismo implementa, capacidades do estado desenvolvido e o papel e peso político do organismo no quadro institucional geral das políticas de juventude desde o estado nacional.

Palavras-chave

Estado, juventude, instituição política, política governamental.

Información autor

[*] Doctor en Política y Gobierno por la Facultad de Ciencia Política y RRII de la UCC, Córdoba, Argentina; magíster en diseño y gestión de programas sociales por la Facultad Latinoamericana de Ciencias Sociales (Flacso, sede Argentina) y licenciado en Ciencia Política por la Universidad Católica de Córdoba (UCC). Actualmente, becario posdoctoral de Conicet. (iD) 0000-0003-4778-7288. Correo electrónico: eduardopereyra_24@hotmail.com 


\section{Introducción}

El trabajo parte del consenso - alcanzado por gran parte de la bibliografía especializada en el tema- de que el concepto de juventud es una categoría histórica que adquiere su especificidad según la construcción social en que se da; es decir, se asocia lo juvenil con un sector poblacional que construye su identidad en el marco de las relaciones sociales e institucionales, las cuales son históricamente construidas y determinadas (Margulis, 2001). A su vez, se considera que no toda acción estatal es resultado de un mero proceso de racionalidad técnica en la toma de decisiones, sino que constituye la instrumentación de un proyecto político determinado (Ander-Egg, 1995). A partir de estas definiciones cabría suponer que la institucionalidad juvenil adquiere particularidades según el contexto político y socioeconómico de un país o territorio y, sobre todo, que estaría condicionada por las concepciones político-ideológicas adoptadas por los distintos partidos gobernantes. Sin embargo, en el caso específico de nuestro análisis sobre los organismos gubernamentales de juventud, este último supuesto de condicionalidad no se observa de manera muy clara. ${ }^{1}$

En primer lugar, conviene aclarar que el artículo hace hincapié en la importancia de las instituciones - en este caso el Estado- y en la influencia que ejercen sobre la definición, la forma de abordar y el despliegue de una temática-problemática (Acuña, 2013). En tal sentido, y teniendo presentes los fines de este trabajo, ${ }^{2}$ se parte de un análisis cronológico y de contenido de fuentes secundarias, principalmente de literatura especializada en la producción estatal de políticas dirigidas a las juventudes a partir de diseños metodológicos cualitativos. Ello con el objetivo de intentar una primera aproximación a lo que los organismos nacionales de juventud vienen produciendo en Argentina tras el

\footnotetext{
${ }^{1}$ Esta observación sobre el caso argentino es la principal razón por la cual se decidió acotar el análisis sobre la institucionalidad juvenil en «sentido estricto». Más adelante se profundiza su distinción.

2 Se recuerda que el trabajo se enmarca en el tipo de artículo revisión de tema, por lo cual esta es una primera exploración -y, por tanto, no acabada - de la literatura. Pero, a la vez, avanzamos en la propuesta de un posible desarrollo teórico interpretativo sobre la institucionalidad juvenil desde la variable proyecto de gobierno.
} 
retorno democrático, en 1983. Los trabajos cualitativos revisados nos permitieron indagar de manera amplia sobre los sentidos, visiones y representaciones que existen sobre la juventud, la cuestión juvenil, el rol del Estado en la materia y el sentido del trabajo institucional efectuado a lo largo de los gobiernos nacionales entre 1989 y 2015.

Como entrada teórica, agregamos al análisis la cuestión ideológica. Mediante la variable proyecto de gobierno, de corte neoliberal o posneoliberal, intentamos comprender si dichos proyectos ejercen influencia sobre la conformación de la institucionalidad juvenil y, por ende, divisar una variación en la performance de estos dos tipos de gobierno.

La lectura crítica-reflexiva de estos trabajos permite observar la existencia o inexistencia en Argentina de (dis)continuidades; estas últimas abren, a su vez, la posibilidad de buscar nuevas variables y tipos de abordaje de la especificidad por los organismos nacionales de juventud.

Puntualmente, sostenemos que el Estado cumple un rol central en la estructuración y configuración de la cuestión juvenil por medio de los distintos organismos y de las políticas públicas que estos implementan. En este sentido, desde finales de la década de los noventa se promueve la superación de los tradicionales esfuerzos del Estado nacional dirigidos a abordar la cuestión juvenil, los cuales tenían como eje central la construcción de espacios específicos. Para ello, se parte del criterio de adoptar un enfoque generacional que atraviese las distintas dependencias estatales y las políticas que implementan. Sin embargo, en Argentina, la institucionalidad estatal continúa desplegando organismos específicos que desarrollan planes, programas y proyectos dirigidos a las juventudes, lo que obliga a seguir focalizando la atención en ellos, a fin de analizar y comprender sus actuaciones.

En función de esto último, más lo expuesto al inicio de esta sección, el presente trabajo se centra analíticamente en el abordaje de la institucionalidad pública juvenil nacional, en sentido estricto. Con esto nos referimos al nivel de la administración que se ocupa específicamente de los problemas de la juventud, es decir, a los organismos gubernamentales de juventud (OGJ), pues entendemos que de este nivel emanan las políticas de juventud, en sentido estricto (Balardini, 1999). En el caso argentino, el OGJ de escala nacional corresponde actualmente al Instituto Nacional de Juventud.

A partir de la exploración del desarrollo institucional realizado por el Estado nacional argentino entre 1989 y 2015 y de sus intervenciones sobre los temas relacionados con la juventud, el objetivo central del trabajo es indagar sobre las (dis)continuidades de las lógicas institucionales para abordar la cuestión juvenil. Para ello se tienen en cuenta los 
tipos de programas y dispositivos diseñados, las capacidades estatales 3 generadas para su implementación y el rol y peso político del organismo en el entramado institucional general de las políticas juveniles promovidas por el Estado nacional.

En función de lo expuesto, el análisis realizado también dialoga con aquellos trabajos que han indagado en la correlación entre los tipos de gobierno y el desarrollo de políticas públicas, particularmente las juveniles (Rodríguez, 2015a, 2018). Por ende, el periodo que escogimos coincide con el desarrollo, en Argentina, de dos proyectos que se distinguen claramente y que en el espectro ideológico se consideran contrapuestos. El periodo comprendido entre 1989 y 2003 ha sido caracterizado como neoliberal, mientras que el que va del 2003 al 2015 se define como posneoliberal (Ramírez-Gallegos, 2012; Sader, 2008). Como veremos más adelante, estas categorías no son antagónicas, sino que con-tienen rupturas y continuidades. Sus diferencias nos permiten suponer que efectivamente habría un tipo de institucionalidad juvenil definido por los proyectos de gobierno a cargo del poder ejecutivo nacional.

El presente artículo se divide en cuatro partes. El primer apartado desarrolla brevemente la emergencia de la cuestión juvenil en la agenda estatal, junto con las principales miradas y recomendaciones que los especialistas y los organismos internacionales hicieron a los Estados para abordar la cuestión juvenil. En el segundo punto desarrollamos la categoría proyecto de gobierno y su potencialidad para estudiar y comprender el desarrollo de la institucionalidad juvenil. En el tercer apartado presentamos una revisión bibliográfica que muestra los contrastes políticos de las gestiones neoliberales (1989-2003) y posneoliberales (2003-2015) en Argentina, al tiempo que señalamos sus diferencias de enfoque, destacamos las rupturas y continuidades en los modelos político-institucionales y las particularidades de lo desplegado por los OGJ durante ambos periodos. Finalmente, en el cuarto punto, exponemos algunas reflexiones desprendidas de esta primera revisión del tema y proponer una agenda futura de líneas de trabajo que contribuyan a la comprensión del desarrollo de la institucionalidad estatal juvenil.

3 Por capacidades estatales se entiende la competencia de los Estados para proveer bienes y servicios, es decir, «mirar hacia dentro del Estado» para comprender cómo este, a partir de recursos materiales y económicos, instrumenta sus objetivos y decisiones políticas (Oszlak, 2004). 


\section{La emergencia de la cuestión juvenil en la agenda estatal y la génesis}

\section{de la institucionalidad específica en los Estados latinoamericanos}

En la década de los ochenta, en pleno contexto de movilización política y recuperación democrática de gran parte de los países del continente americano, y teniendo presente que las juventudes fueron el principal grupo poblacional que sufrió las consecuencias más crudas de las crisis político-económicas del momento, así como la combinación de tres hitos institucionales de carácter internacional (Beretta et al., 2018), se impulsó el ingreso de la cuestión juvenil en la agenda pública de los Estados latinoamericanos. Como señalan estos autores, los hitos se refieren, en primer lugar, a la celebración en 1985 del Año Internacional de la Juventud (AIJ), declarado por la Asamblea General de Naciones Unidas en 1979. Se buscaba movilizar y promover la formulación de políticas estatales que garantizaran los derechos humanos de las y los jóvenes. El segundo hito es la Jornada Mundial de la Juventud, instituida por la Iglesia católica en el marco del AIJ, y cuyo segundo encuentro, en 1987, se realizó en Argentina, coincidiendo con la conformación de la Subsecretaría Nacional de la Juventud. Este último es considerado por autoras como García y Liguori (2015) como el primer organismo nacional sectorial dedicado específicamente a atender la cuestión juvenil en el país. Finalmente, y de manera significativa para América Latina, se identifican las conferencias intergubernamentales sobre políticas de juventud en Iberoamérica celebradas a finales de los años ochenta y la fundación de la Conferencia Iberoamericana de Ministros de la Juventud, a inicios de los noventa, que luego se transformaría en la Organización Iberoamericana de la Juventud (OIJ), instancias promotoras de las políticas juveniles estatales.

En definitiva, este conjunto de sucesos político-institucionales propició el ingreso de la cuestión juvenil en la agenda estatal, el desarrollo de una institucionalidad mediante la creación de organismos especializados, la implementación de políticas juveniles sectoriales y la promulgación de leyes (e, incluso, reformas en el sistema judicial). Según Dávila-León y Silva (1999), en aquel momento parecía

haber una suerte de acuerdo en orden a convenir que la existencia de una política pública de juventud sin una necesaria institucionalidad pública en materia de juventud, no [era] algo sustentable en el tiempo. La institucionalidad es quien puede aportar las bases de permanencia y continuidad de determinadas políticas públicas. (p. 6)

Uno de los grandes debates sobre el tema, y particularmente respecto al papel del Estado en la cuestión juvenil, ha girado en torno a la conveniencia o inconveniencia de 
la conformación de organismos gubernamentales específicamente dedicados a los temas relacionados con las juventudes, su articulación con la política sectorial y la distribución de roles en el plano multinivel.

En los inicios, la academia y los organismos internacionales aconsejaron que los temas de las juventudes fueran abordados específicamente por organismos gubernamentales especializados en el tema (OGJ), instituciones que debían funcionar en los distintos niveles estatales. A la vez, se esperaba de cada nivel una distribución funcional de roles estratégicos: la nación sería la responsable de «dinamizar los procesos de concertación interinstitucional, cumpliendo papeles de animación, articulación y sensibilización, mientras que [...] [provincias y municipios] deberían responsabilizarse por la ejecución efectiva de programas y proyectos» (Comisión Económica para América Latina y el Caribe [Cepal] \& OIJ, 200o, pp. 24-25). A partir de los años noventa, en toda Latinoamérica se extendió la decisión de los Estados nacionales de generar una institucionalidad específicamente enfocada en los temas de la juventud. En consecuencia, se crearon estructuras burocráticas con organismos exclusivos para abordar los temas y problemas de las y los jóvenes. Algunos países reforzaron la institucionalidad con leyes nacionales de juventud que garantizaran la creación y permanencia de dichas instituciones o sancionaron una normativa que establecía derechos y obligaciones del sujeto joven. ${ }^{4}$

En proximidades del nuevo siglo se revisó críticamente esta estrategia estatal latinoamericana de abordaje de los temas juveniles a partir de los resultados e impactos de las políticas implementadas por los OGJ y, principalmente, de las debilidades institucionales-organizacionales que se detectaron en dichos organismos. Sobre esto último, se ha señalado como característica general la incapacidad de los organismos para lograr que los ministerios incluyeran puntos de su agenda en sus programas, así como problemas de distribución de competencias. Cuando los OGJ se proponían abordar cuestiones sectoriales de su agenda (como salud, educación o empleo), terminaban perdiendo frente a las propuestas de las grandes secretarías de Estado o ministerios. Además, estos últimos, en los programas que diseñaban - y en su implementación - aplicaban enfoques conservadores y adultocéntricos, manejando conceptos estereotipados de las juventudes; con ello demostraban un gran desconocimiento de la dinámica juvenil en la realidad sobre la cual intentaban actuar (Rodríguez, 2003).

4 En términos generales, estos son algunos casos que optan por una o ambas opciones: Chile (1991), Colombia (1997 y 2006), México (1999), República Dominicana (2000), Ecuador (2001), Nicaragua (2001), Perú (2002 y 2005), Costa Rica (2002), Venezuela (2002) y Brasil (2005). Para analizar con mayor especificidad véanse Borzese et al. (2009), así como Krauskopf (2008). 
Por estos motivos, a principios del siglo XXI se propuso priorizar la incorporación de una mirada generacional como un nuevo enfoque aplicable al conjunto de políticas públicas:

Importa dotar a las políticas públicas en su conjunto de una perspectiva generacional, superando resueltamente el enfoque - acotado - de trabajo prevaleciente hasta el momento (sectorializado, monopólico, centralizado, etc.), evitando caer en los espacios y programas exclusivos para adolescentes y jóvenes como hasta el momento (que solo han reforzado el aislamiento de los jóvenes) y tratando de incorporar estas temáticas particulares a todas y cada una de las políticas públicas, emulando a la perspectiva de género impulsada por las mujeres, que ha tenido más y mejores resultados, en la mayor parte de los países de la región. (Rodríguez, 2003, p. 31)

Desde esta nueva óptica, lo principal ya no era necesariamente desarrollar un OGJ como condición para abordar los temas de la juventud en cada nivel estatal, sino formar a toda la estructura burocrática y de funcionarios políticos encargados del diseño e implementación de políticas públicas juveniles (directas e indirectas) para que comprendiera a las juventudes desde su heterogeneidad, como un sujeto social con necesidades y derechos específicos. El objetivo principal era derribar los mitos y prejuicios del adultocentrismo sobre la condición juvenil. Dicha postura mayoritariamente la entiende como una «etapa de transición del ciclo de vida», sobre la cual el Estado tiende a generar mecanismos para controlar el desarrollo de pautas y valores dirigidos a garantizar su reproducción social.

Sin embargo, Rodríguez (2018) señala que, a diferencia de los grandes avances logrados en materia de perspectiva de género - y a más de quince años de esta propuesta generacional-, en el conjunto de políticas públicas son pocas las experiencias de gestión de asuntos de la juventud que han logrado una mirada integral, transversal, coherente y con enfoque de derechos. Además, lejos de desaparecer, los OGJ (en los ámbitos estatal, nacional y provincial) continúan vigentes en la mayoría de las estructuras orgánicas estatales latinoamericanas y su creación se está extendiendo cada vez más en los niveles locales de gobierno.

Hay una producción estatal de políticas, proyectos y programas sobre las juventudes que se genera en las OGJ y que definen un modo de entender la realidad de la cuestión juvenil en cada territorio. De allí la importancia de abordar la institucionalidad específica, para debatir y reflexionar sobre sus características y su potencial vínculo con los proyectos de gobierno, finalidad central de este artículo. 
Por último, Krauskopf (2008) identifica dos grandes tendencias que han primado en la estructuración de los organismos oficiales que manejan los temas de la juventud:

A. Organismos de juventud que carecen de personería jurídica propia, adscritos a un ministerio no especializado en el tema o a unidades administrativas menores que no tienen peso ni son prioritarias para los futuros planes de gobierno. De allí que se vean muy limitados en sus despliegues administrativos y políticos.

B. Organismos oficiales de juventud adscritos a una institución o dependencia estatal de alta jerarquía (incluso puede ser especializada), que en ocasiones pueden contar con autonomía administrativa y política. Para ellos es clave la concreción de un vínculo directo con un órgano con poder político y con fuerte potencialidad en el abordaje intersectorial y transversal de los temas relativos a la juventud.

A continuación analizaremos la categoría proyecto de gobierno y sus clasificaciones para comprender las definiciones políticas e ideológicas de un gobierno y los provisorios vínculos que diversos estudios sobre juventudes señalan entre esta categoría y la configuración de un determinado tipo de política juvenil (en términos amplios). Asimismo, buscaremos observar cómo esta discusión es conceptualmente pertinente y útil para focalizar el análisis de una institucionalidad juvenil específica.

\section{El vínculo entre proyectos de gobierno y tipos de políticas públicas juveniles en Latinoamérica}

Peter Hall introduce el concepto de policy paradigms, es decir, marcos interpretativos que moldean objetivos y estrategias de los agentes estatales, como lo son el keynesianismo y el neoliberalismo. Estos paradigmas son defendidos tanto por políticos y burócratas como por el conjunto de la sociedad, operando de distintos modos, según el caso (Viguera, 2000). Como resalta Stoessel (2014), en el momento de definir los gobiernos, debemos «partir de los contextos específicos y prácticas y agendas políticas que le dan sentido, y no a partir de valores ahistóricos que esencializan los proyectos políticos descontextualizados de su realidad inmediata» (p. 39).

Desde finales de la década de los noventa se viene señalando que los gobiernos nacionales de América Latina han realizado un «giro a la izquierda» (Nazareno, 20o9; RoviraKaltwasser, 2011; Stokes, 2008;), fundamentalmente si sus políticas se contraponen con los planes de ajuste estructurales-neoliberales del Consenso de Washington, impuestos en el continente desde mediados de los años setenta. Para el 2008, el 65\% de la población 
de la región estaba siendo gobernada por el arco progresista, ya que en 11 de los 18 países latinoamericanos se instauraban proyectos posneoliberales (Stokes, 2008; Nazareno, 2009), cuyas experiencias adoptaban denominaciones tales como «socialismo del siglo XXI», «revolución ciudadana», «neodesarrollismo»o «modelo nacional y popular».

En el caso particular de Argentina, hay un amplio consenso en que hubo un gobierno posneoliberal durante el kirchnerismo (2003 y 2015), sobre todo si se lo contrapone con lo sucedido en la década neoliberal, principalmente en los gobiernos de Menem (1989-1999) y De la Rúa (1999-2001). Diversos autores definen los gobiernos de Néstor Kirchner (2003-2007) y de Cristina Fernández de Kirchner (2007-2015) como posneoliberales. A pesar de las diferentes denominaciones con las que se ha etiquetado el periodo (como «neodesarrollista»; Bresser-Pereira, 2007; «reformista»; Ramírez-Gallegos, 2012; «moderado»; Alegre, 2008; «nacional y popular»; Rey, 2014; o en términos negativos, como «izquierda populista»; Castañeda, 2006), en el mundo académico y político existe un vasto acuerdo sobre que, en ese periodo, el país participó en el giro a la izquierda que se observó en Latinoamérica. Para Laguado y Cao (2015), la disputa central de estos gobiernos giró en torno a la cuestión del poder y a los conceptos de igualdad y justicia social. La política de desarrollo de Néstor Kirchner se basó en la (re)industrialización, apostando por el círculo virtuoso keynesiano de pleno empleo, mercado interno, fortalecimiento fiscal, inversión social y obtención de superávit fiscal, al tiempo que se mantenía un tipo de cambio competitivo. Con una estrategia mixta, el Estado asumió un papel preponderante en la inversión tomando, en algunos casos, el control total de empresas de sectores estratégicos y, en otros, un control parcial y compartido con el sector privado.

Sin embargo, a partir de diversos procesos destituyentes caracterizados como neogolpismo o golpes blandos, 5 como los casos de Aristide en Haití (en 2004), de Zelaya en Honduras (en 2009), de Lugo en Paraguay (en 2012) y de Dilma Rousseff en Brasil (en 2016), asistimos a un nuevo giro político, esta vez hacia la derecha. Estos casos tuvieron la particularidad de que las nuevas presidencias se produjeron a partir de la desestabilización política mediante manifestaciones callejeras, y no por intervención de cuarteles militares. Estos procesos destituyentes se gestaron con la articulación política de movimientos y partidos políticos conservadores con los medios dominantes y facciones del poder judicial. A esta nueva ola de derecha hay que agregar los triunfos electorales de los empre-

5 «El "nuevo golpismo" está encabezado más abiertamente por civiles y cuenta con el apoyo tácito (pasivo) o la complicidad explícita (activa) de las Fuerzas Armadas, pretende violar la constitución del Estado con una violencia menos ostensible e intenta preservar una semblanza institucional mínima (por ejemplo, con el Congreso en funcionamiento y/o la Corte Suprema temporalmente intacta» (Tokatlian, 2009). 
sarios Cartes (en Paraguay en 2013), Varela (en las presidenciales de Panamá en 2014), Macri (en Argentina en 2015), el regreso de Piñera al poder en Chile (en 2017) y el triunfo del ultraderechista Jair Bolsonaro en Brasil (2018).

Para Giordano (2014), esta nueva derecha compite incluso programática y discursivamente con los gobiernos progresistas, ya que ha retirado de la agenda propuestas reprivatizadoras o impulsa propuestas de mayor inclusión.

Esta discusión nos indica que en América Latina las categorías izquierda y derecha siguen vigentes teórica y prácticamente (Lievesley, 2009), pero que se han reconfigurado respecto a sus categorizaciones clásicas, anteriores a los años ochenta. Bobbio (1995) señala tres aspectos centrales en esta distinción de derecha e izquierda: son conceptos antitéticos, es decir, que uno existe gracias al otro; la distinción medular se basa en la concepción que cada una tiene de la igualdad (para la derecha, las desigualdades son naturales y, por ende, difíciles de erradicar, mientras que para la izquierda son socialmente construidas); por último, se distinguen por la actitud que toman sobre la igualdad (para la derecha, el Estado debe garantizar igualdad de oportunidades, mientras que la izquierda propone la búsqueda de igualdad de resultados).

Para definir los proyectos de gobierno utilizamos las categorías neoliberal y posneoliberal ${ }^{6}$ (Ramírez-Gallegos, 2012; Sader, 2008). Los neoliberales se asocian con modelos de reformas estatales y gestión desarrollados en los noventa, vinculándose políticamente con la derecha. Dan mayor preponderancia al mercado, en detrimento de la intervención activa del Estado en la producción del bienestar de la población. El segundo modelo, vinculado a gobiernos progresistas o de nueva izquierda, aspira articular crecimiento económico y redistribución social, al tiempo que propugna una inclusión sociopolítica que combina diversos formatos democráticos y aspira a la vuelta de la centralidad del Estado (Stoessel, 2014).

Como resultado de los estudios y análisis de la experiencia latinoamericana, los trabajos ya citados coinciden en afirmar que los distintos tipos de proyectos de gobierno - dependiendo de su configuración político-ideológica- estructuran una particular agenda pública con respuestas diferenciadas, por medio de políticas y modelos institucionales. La naturaleza neoliberal o posneoliberal del gobierno tiene consecuencias en el despliegue institucional, los roles estatales asumidos y la redistribución del bienestar.

\footnotetext{
${ }^{6}$ No obstante, el posneoliberalismo no es antagónico al neoliberalismo en la medida en que hasta el momento no constituyen un quiebre del sistema capitalista, sino que más bien se cobijan importantes rupturas y algunas continuidades con este.
} 
Toda política pública guarda una relación básica de consistencia con los diseños estratégicos que orientan el ejercicio del poder político. El modo en que las políticas son producidas y ejecutadas, sus objetivos específicos o sectoriales, los recursos que se les destinan, siempre son referibles, directa o indirectamente, a esos diseños, que adquieren expresión institucional, formal e informal, en el régimen político. (Vilas, 2012, p. 45)

Un proyecto de gobierno, sin importar cuál sea su tipo, adopta un modelo de acumulación y desarrollo; luego selecciona y problematiza una serie de cuestiones que incluirá en su agenda pública (Aguilar-Villanueva, 1993); y, finalmente, determina qué se va a producir, cómo y para quiénes. Estarán planificados tanto la obtención de recursos como su utilización y la distribución de la riqueza generada.

Particularmente, sobre el vínculo entre los tipos de gobierno y el desarrollo de políticas de juventud en América latina, Rodríguez (2015a) ha realizado una interesante primera exploración. De forma similar a la que venimos desarrollando, el autor ha clasificado los tipos de gobierno en neoliberales y neodesarrollistas (estos últimos corresponderían a los posneoliberales, según nuestro criterio) ${ }^{7}$ y distribuye en ellos los distintos países de Latinoamérica según su matriz política.

Para hallar el posible vínculo entre el proyecto de gobierno y el tipo de políticas juveniles diseñadas e implementadas por cada país según su proyecto de gobierno, el autor se propone buscar evidencias a partir de la aplicación de tres indicadores: el índice global de desarrollo juvenil, el índice de desarrollo de políticas integradas de juventud y los resultados del estudio de la inversión social en juventud.

A continuación, exponemos una breve reseña de los resultados obtenidos por Rodríguez (2015a) sobre cada indicador, con sus principales conclusiones:

A. Indice global de desarrollo juvenil. Es aplicado por las Naciones Unidas para evaluar los impactos del Plan de Acción Mundial de Juventud desde su implementación en 2005. Aborda cuestiones «objetivas» (salud, educación y empleo) y «subjetivas» (participación ciudadana y política). En materia de educación, por ejemplo, analiza el porcentaje del PIB invertido en educación y los años de educación y alfabetización juvenil. En materia de salud registra la tasa de mortalidad juvenil, la tasa de embarazo y el consumo de tabaco,

\footnotetext{
7 Respecto a la clasificación, nuestro estudio contempla tanto el neodesarrollismo como el neokeynesianismo/ socialdemócrata/socialismo/populismo radical como tipos de manifestación de la categoría más amplia de gobiernos posneoliberales. Para profundizar sobre este aspecto, véanse los trabajos de Ramírez-Gallego (2012) y Sader (2008).
} 
entre otros factores. En cuanto al empleo, indaga sobre el peso de la población juvenil en la tasa total de empleo y el desempleo juvenil. En lo que respecta a la participación política y cívica, indaga sobre la representación juvenil y educación electoral para la primera, y las acciones de voluntariado, para la segunda. A partir de las ponderaciones aplicadas, queda en evidencia que nueve de los países neoliberales cuentan con un desarrollo de nivel medio (con un promedio de o.61 puntos) y uno con nivel bajo. En cuanto a los gobiernos neodesarrollistas, ocho cuentan con un nivel medio (con un promedio de 0.65 puntos) y dos con altos niveles de desarrollo. Comparativamente, parecería haber una pequeña ventaja del desempeño de los países neodesarrollistas sobre los neoliberales.

B. Indice de desarrollo de políticas integradas de juventud. Lo viene desarrollando el Centro Latinoamericano sobre Juventud (Celaju) y contempla principalmente quince variables de índole institucional (existencia o inexistencia de un instituto gubernamental de juventud, de una ley general de juventud, un consejo de juventud, encuesta nacional de juventud, estadísticas sobre juventud, sistema de monitoreo y evaluación de políticas) y otras variables de naturaleza programática (como la existencia de una política nacional y un plan de acción nacional de juventud). Tras asignar un puntaje a la existencia o carencia del indicador y al grado de desarrollo - en caso de que exista-, dicha herramienta establece un escalafón en el que ubica a cada país. Según este índice, los países neoliberales tendrían una leve ventaja (promedio total de 11.1/45 puntos) sobre los neodesarrollistas (promedio total de $10 / 45$ puntos).

C. Inversión social en juventud. Este estudio pertenece a la Comisión Económica para América Latina y el Caribe y la OIJ. Calcula cuantías de inversión en educación, salud, protección social y vivienda, al tiempo que compara la inversión real con lo que debería invertirse si se considerara el peso demográfico de las juventudes. Los países se clasifican a partir de las cuantías de inversión detectadas. En este tienen mejor desempeño los gobiernos neodesarrollistas (promedio de o.66 puntos) que los neoliberales (promedio de 0.55 puntos), aunque en cada grupo se encontraron países con altos y bajos grados de inversión.

Rodríguez considera que los resultados permiten constatar ciertas diferencias en el desempeño en políticas de juventud, según el tipo de gobierno de un país, pues se reconoce una mejor actuación de los neodesarrollistas que de los neoliberales en el plano sectorial y en la proporción de la inversión. Además, a modo de control de este vínculo, el mismo autor en otro trabajo (Rodríguez, 2015b) analiza la posible relación entre crecimiento económico y desarrollo humano, por un lado, y desarrollo de políticas de juventud, 
por otro, y no halla correlaciones consistentes. Por esto, la relación con los proyectos de gobierno se manifiesta como el vínculo que se debe seguir explorando. ${ }^{8}$

Consideramos que el análisis explicativo de la correlación entre los proyectos de gobierno y la estructuración y el despliegue de políticas juveniles tiene un interesante potencial. No obstante, nuestro foco de atención se circunscribe, en esta inicial aproximación, solo al desarrollo de institucionalidad juvenil específica. Rodríguez (2015a) contempla los aspectos de institucionalidad en las variables institucionales del índice de desarrollo de políticas integradas de juventud; indica que, en la medición de la correlación, la balanza se inclina en favor de los gobiernos neoliberales, que son los que más atención prestan al desarrollo de herramientas institucionales, por lo cual dispondrían de un mejor desempeño en el despliegue de institucionalidad juvenil.

Sin embargo, a partir de nuestra primera exploración descubrimos que, en el caso argentino, una correlación entre proyecto de gobierno y los aspectos institucionales específicos de los OGJ no es tan clara. Nuestro análisis cualitativo de la información aportada por fuentes secundarias pone en evidencia que tanto los gobiernos neoliberales como los posneoliberales comparten un muy escaso desarrollo de institucionalidad específica; con lo cual la relevancia de los aspectos ideológicos (en la categoría proyecto de gobierno), como variable explicativa del desarrollo de un tipo de institucionalidad, se ve relativizada.

A continuación exponemos las principales características sociopolíticas y económicas de los proyectos de gobierno vigentes entre 1989 y 2015, que permiten calificar de neoliberal lo desplegado entre 1989 y 2003, y de posneoliberales los gobiernos kirchneristas que se sucedieron entre 2003 y 2015. A la vez, se recuperan estudios que permiten señalar las principales (dis)continuidades institucionales que los organismos nacionales de juventud experimentaron durante ambos periodos.

\section{V'́nculos entre proyecto de gobierno e institucionalidad juvenil específica en Argentina: una historia de rupturas y continuidades}

En el presente apartado profundizaremos sobre las principales características de la institucionalidad juvenil nacional entre 1989 y 2015. Elegimos este lapso porque la bibliografía especializada identifica los proyectos de gobierno ocurridos entre 1989 y 2003

8 Dado el carácter incipiente del hallazgo, el autor considera que aún se deben profundizar, complejizar y ajustar las metodologías, las ponderaciones y los indicadores utilizados. 
como de fuerte corte neoliberal, mientras considera posneoliberales los gobiernos existentes entre 2003 y 2015. Esta distinción nos permitió avanzar exploratoriamente en nuestro análisis a fin de estudiar de manera comparada si durante estos tipos de proyectos de gobierno los OGJ adquirieron o no una especificidad diferenciadora en su institucionalidad; además, si esta podría ser comprendida por un vínculo con la categoría ideológica propuesta, cuya potencialidad fue destacada en el apartado anterior. Para tales fines se prestó especial atención a aspectos como las principales características de las lógicas de abordaje de la cuestión juvenil implementadas por los programas y dispositivos del OGJ, las capacidades administrativas desarrolladas por este y el rol y peso político del organismo en el entramado institucional general de las políticas juveniles puestas en marcha por el Estado nacional.

\section{El Organismo Nacional de Juventud durante el proyecto neoliberal (1989-2003)}

Desatada la crisis mundial del Estado social y su modelo de acumulación y crecimiento, se propuso realizar una nueva reforma del Estado. ${ }^{9}$ Esta, como bien sostiene Fleury (2000), no consistió en una simple reforma administrativa: fue una transformación de las relaciones de poder, del modelo de desarrollo e inserción en la economía internacional e, incluso, comprendió una reconfiguración de la hegemonía del bloque dominante.

Para América Latina, y en particular para Argentina, el proceso de reforma fue de claro corte neoliberal. El ajuste fiscal y el desmantelamiento estatal fueron los criterios que predominaron. Sin embargo, para garantizar este nuevo modelo fue necesaria una intervención activa del gobierno a cargo del Estado para sancionar nuevas normativas, avanzar en determinados «autodesmantelamientos» y estructurar el nuevo orden de acumulación.

Luego de las consecuencias de las fallidas reformas de primera generación, ${ }^{10}$ con las que se intentó reducir no solo el tamaño, sino también el rol del Estado, los propios organis-

\footnotetext{
9 Según Oszlak (1990), la reforma del Estado atendió cuatro problemas: dos de carácter económico-político (la delimitación del tamaño del Estado y la redefinición del papel regulador del Estado), un problema de carácter económico-administrativo (relativo a la recuperación de gobernancia) y un dilema político. El eje fundamental de la reforma fue la gobernabilidad.

${ }_{10}$ Nos referimos a las reformas impulsadas por los organismos financieros internacionales en América Latina desde finales de los ochenta hasta la primera mitad de la década de los noventa. Implicaban un achicamiento del Estado y el abandono de sus funciones de productor de bienes y servicios para abordar la cuestión social y el desarrollo de bienestar. Las estrategias más comunes que adoptaron fueron la privatización, la tercerización, la descentralización, el ajuste fiscal, la disminución de la inversión social y la liberalización económica.
} 
mos multilaterales que recomendaron esas reformas reconocieron que menos Estado no implicaba necesariamente uno mejor. Se impulsaron entonces las reformas de segunda generación, las cuales pusieron en la agenda pública la discusión sobre cómo mejorar el desempeño del Estado, su funcionamiento administrativo, su poder regulatorio, la producción eficiente y eficaz de los resultados propuestos por las políticas públicas implementadas. El desafío propuesto consistía en desarrollar y optimizar la capacidad estatal a partir de la incorporación del paradigma de la nueva gerencia pública, ${ }^{11}$ aunque este objetivo de mejorar la calidad institucional quedó «a mitad de camino»(Oszlak, 1999).

Como consecuencia, aquella Argentina que se destacaba en el continente por sus menores niveles de pobreza, indigencia y disparidad en la distribución del ingreso, así como también por un sólido mercado de trabajo, tendiente al pleno empleo, dejó de existir con la consolidación del proyecto neoliberal en este periodo.

En términos del rendimiento de las políticas públicas y su relación directa con las consecuencias negativas del proceso de reforma del Estado nacional, las respuestas de las instituciones responsables de las políticas relacionadas con la juventud fueron ineficientes e ineficaces. Rodríguez (2000) afirma que, durante los noventa, los errores derivaron principalmente de los paradigmas neoliberales adoptados para comprender a las y los jóvenes y la consecuente configuración de determinados «modelos» de políticas de juventud: el más antiguo se centra en la educación y el tiempo libre de las y los jóvenes; los centrados en el control social de jóvenes movilizados y de la pobreza como método para combatir el delito; y, desde mediados de los noventa, el modelo de inserción laboral juvenil basado en la «inversión del capital humano». Entre las características comunes de estos modelos, el autor menciona la pretensión de universalidad de las políticas y la excesiva burocratización del Estado para desarrollarlas.

Las políticas específicas del organismo gubernamental juvenil apuntaban a la preparación de las personas jóvenes para su ingreso en el mundo adulto mediante la educación, para lo cual se funcionalizaba «adecuadamente» la utilización del tiempo libre y se definía a la juventud como «la esperanza del futuro». Además se señala que, al contrario de las claras urgencias sociales juveniles que existían en ese contexto (como la pobreza y exclusión), gran parte de las políticas eran destinadas a las y los «jóvenes integrados» (jóvenes estudiantes o de clase media) (Rodríguez, 200o).

\footnotetext{
${ }_{11}$ De marcado corte promercado, los componentes que la conforman suponen: a) flexibilidad de la organización estatal; b) legitimidad basada en la eficiencia económica, y c) centralidad del manager, o gerente, en la gestión de los recursos humanos (Osborne \& Gaebler, 1992).
} 
En cuanto a las capacidades administrativas y al rol político-institucional del OGJ, el trabajo de Balardini y Hermo (1995) expone sus profundas debilidades:

- Nula legitimación política y social de la institucionalidad «política de juventud».

- Baja sistematicidad y organicidad de la Institucionalidad estatal sobre juventud, determinada por los avatares políticos de cada gobierno.

- Bajo presupuesto.

- Objetivos y políticas cortoplacistas.

- Falta de estudios y diagnósticos respecto a la realidad juvenil argentina, generando mucha improvisación.

- Falta de recursos humanos especializados en el tema.

- Falta de seguimiento y evaluación de programas implementados.

- Falta de coordinación y articulación de los organismos estatales para diseñar y ejecutar políticas juveniles.

- Las políticas implementadas no les dan participación a las y los jóvenes y organizaciones vinculadas a jóvenes, principales destinatarios de las mismas.

- No existe ninguna institución federal que nuclee los organismos de juventud del país para coordinar y articular esfuerzos.

- Inexistencia de legislación nacional que les dé sistematicidad y continuidad a las políticas nacionales de juventud. (Balardini \& Hermo, 1995, p. 51)

En definitiva, durante el proyecto neoliberal la institucionalidad estatal juvenil, en sintonía con el desmantelamiento del rol y el despliegue institucional, se destacó por un bajo grado de desarrollo y desempeño (Oszlak, 1999). Sin embargo, conviene prestar mucha atención a esta caracterización, puesto que veinte años después, y habiéndose desarrollado un nuevo proyecto de gobierno entre el 2003 y el 2015 de carácter posneoliberal, la institucionalidad juvenil, en sentido estricto, pareció reproducir la misma tendencia.

En cuanto al devenir de los organismos juveniles en la estructura orgánica interna del Estado, se detectó que han mantenido un patrón similar en el transcurso de la historia, vinculados a dependencias que orbitan en torno a estructuras no específicas, lo cual atenta contra sus posibilidades de desplegar una amplia autonomía administrativa y política, identificándolos con la primera categoría de organismos, según la clasificación de Krauskopf (2008). En cuanto a su historicidad, el primer antecedente surge con la creación del Área de Juventud, en 1985, en el marco del Año Internacional de la Juventud, que luego, en 1987, se convierte en una subsecretaría dependiente de la Secretaría de Desarrollo Humano y Familia del Ministerio de Salud y Acción Social. En 1989, el organismo es remplazado por la Dirección Nacional de Juventud, que al año siguiente pasa a 
ser Secretaría (Decreto PEN n.. 283 del 19/2/90), luego Área de Juventud, y posteriormente, hasta 1993, Instituto Nacional de Juventud (Decreto PEN n.ํ 1348/90). En esta última fecha es remplazado por la Subsecretaría de Juventud (creada por el Decreto n.․․ 2009/ 93), dependiente de la Secretaría de Asuntos Institucionales del Ministerio del Interior.

En 1996 la subsecretaría vuelve a convertirse en dirección nacional; dos años más tarde nuevamente es transformada en subsecretaría, dependiente de la Secretaria de Relaciones con la Comunidad, del Ministerio del Interior y, en el 20oo, retorna a su condición de Dirección Nacional de Juventud (Dinaju), dependiente del Ministerio de Desarrollo Social y Ambiente; como tal se mantendrá hasta el 2015, cuando se transformó en subsecretaría (SSJ). Finalmente, en 2018 se transformó en el Instituto Nacional de Juventud, sin dejar de pertenecer al mismo ministerio.

\section{La cuestión juvenil en el nuevo contexto de acumulación en Argentina (2003-2015)}

Los gobiernos kirchneristas que se sucedieron a partir del 2003, con las gestiones de Néstor Kirchner (2003-2007) y Cristina Fernández de Kirchner (2007-2011 y 2011-2015), desplegaron nuevas estrategias sociales que modificaron y profundizaron el sistema de protección social de las y los argentinos. ${ }^{12}$ Entre el 2003 y el 2008 se registró un crecimiento económico sostenido a una tasa promedio del $9 \%$, que se vio plasmado en los avances y mejoras del mercado de trabajo, como la disminución de más de la mitad de desocupados (de 3 a 1.4 millones de personas), la reducción de 8.5 puntos en la tasa de empleo no registrado, la recuperación y crecimiento del salario medio real, la reducción del $73 \%$ de la pobreza, mejoras en la distribución del ingreso y el avance de la protección social. Es decir, una matriz de bienestar caracterizado por la reestatización de los fondos de pensiones, el fortalecimiento y creciente protagonismo de las áreas de gobierno central como rectoras de los sistemas de salud y educación y una tendencia a la universalización de la seguridad social (Repetto \& Masetto, 2011, p. 22). Este camino, transitado desde 2003 al 2015, implicó que el Estado nacional adoptara una recuperación de su papel de administrador de la cuestión social argentina, en sintonía con las características propias de una estatalidad impulsada por un proyecto posneoliberal, tal como se definió en el apartado anterior.

${ }_{12}$ Un análisis detallado del conjunto de normas, políticas públicas e institucionalidad desarrollado en el periodo se encuentra en el texto De la sociedad de beneficencia a los derechos sociales (Golbert, 2010). 
En comparación con las estrategias desplegadas durante los noventa, las gestiones kirchneristas reconstruyeron la política social mediante una combinación del enfoque del seguro social con el de derechos. El cambio no solo produjo nuevos paradigmas, sino que se tradujo en una importante reestructuración del diseño institucional estatal para implementar las políticas, así como en la creación de nuevos cuerpos normativo-legales y en un aumento significativo del presupuesto destinado a este tipo de políticas sociales (Tirenni, 2013). El conjunto de políticas del nuevo modelo de acumulación kirchnerista ha hecho que estos gobiernos sean definidos como un proyecto posneoliberal (entre otros, Nazareno, 2009; Sader, 2008; Stoessel, 2014).

Sobre este mismo periodo, algunos autores observan que desde el año 2003 existió un creciente protagonismo de la cuestión juvenil y un notable «reverdecer» de la participación política de las y los jóvenes a partir de su repolitización; no obstante, con algunas instancias más «tradicionales», en la medida en que esta se encauzó principalmente hacia la política partidaria e institucional (Bonvillani et al., 2008).

Sin embargo, cabe remarcar que, a pesar de este auge de lo juvenil, los cambios en el modelo de acumulación, la adopción de nuevos paradigmas (como el enfoque de derechos en sus políticas) y los enormes montos invertidos en la cuestión social y juvenil (es decir, un nuevo tipo de proyecto de gobierno) no se verían reflejados en la creación de una nueva matriz de institucionalidad juvenil específica que operara en el ámbito nacional.

Borzese et al. (2009), en un análisis que abarca fundamentalmente el gobierno de Néstor Kirchner, observan la persistencia de una serie de problemáticas que se sucedieron en la década de los noventa, relacionadas con su discontinuidad y cortoplacismo. En este sentido, y si bien se desplegó una notable batería de políticas transversales y sectoriales al margen del OGJ (principalmente en educación, salud y trabajo), con pretensiones de alcance universal, ${ }^{13}$ estas carecieron de un eje común y articulador. Estos autores también destacan que el OGJ tuvo escasa capacidad para promover y generar investigaciones, conocimientos e información sobre la realidad juvenil, algo que los aportes de los actores de la sociedad civil no lograron compensar, ya que el grado de desarrollo del campo juvenil es aún incipiente en el país. Los autores recalcan, además, el bajo grado de formalización normativa y de institucionalización, ya que durante este periodo todos los organismos específicos surgieron a partir de decretos del poder ejecutivo nacional. El

${ }_{13}$ Criterio claramente diferenciado al de la focalización, propiamente desarrollado en el auge neoliberal de los noventas (Oszlak, 1999). 
despliegue del OGJ dependió en gran medida del contexto político y de la voluntad política del gobierno.

Entre las principales debilidades de la actuación institucional de la Dinaju durante este periodo, los autores resaltan negativamente

el bajo poder político del organismo, que lleva a un nulo poder de injerencia sobre las políticas transversales o de articular de manera conjunta; que no se ha definido una clara línea política para abordar lo juvenil y, por ende, carece de cualquier tipo de continuidad, siendo «desarmada» en cada cambio de gestión, y que su escaso poder incide directamente con el bajísimo presupuesto que maneja, dependiendo exclusivamente de la decisión del ministro a cargo. (Borzese et al., 2009, pp. 64-65)

Del análisis se desprende que, además del escaso vínculo y articulación de la institucionalidad específica con el conjunto de organismos sectoriales y sus políticas juveniles implementadas, hay una decisión política del gobierno kirchnerista de que sea justamente la política sectorial la que tenga mayor preponderancia e injerencia en el abordaje de la cuestión juvenil, y no el OGJ. ${ }^{14}$

Finalmente, podemos destacar los trabajos de Melina Vázquez (2014, 2015a, 2015b) y Núñez et al. (2015) que, por un lado, abordando la relación entre activismo político juvenil y trabajo en el Estado y, por otro, analizando las organizaciones juveniles kirchneristas y el modo como la Dinaju implementó sus programas durante las dos presidencias de Cristina Fernández de Kirchner, nos permiten observar y deducir algunas particularidades de la institucionalidad juvenil del OGJ durante ese periodo.

En términos generales, se advierte que la dirigencia política interpretó la juventud y los temas con ella relacionados como un espacio de vacancia política y una condición para el recambio político en el kirchnerismo. La categoría joven se convirtió en una fuente de prestigio y fue destacada en el universo militante.

En el caso puntual del OGJ (se trate de la Dinaju o de la SSJ), los autores señalan que la pertenencia a determinada organización política habilita los ingresos laborales a dicho organismo, los ascensos y la designación de altos cargos en el organigrama. Esta situación no dista mucho de lo que pasaba en el periodo neoliberal, cuando los nombramientos dependían ostensiblemente del vínculo político y, a la vez, funcionaban

${ }_{14}$ Los motivos de esta decisión política exceden los propósitos del presente artículo, que se limita a una revisión del tema. 
como «trampolín» de ascenso en la jerarquía estatal y política para quienes pasaban por los cargos de mayor relevancia en el organismo.

Vázquez (2015b) afirma que el cambio y ascenso jerárquico de una dirección hacia una subsecretaría nacional, con el consecuente cambio de autoridades, no respondió a una reestructuración del organigrama con el interés de dotar de mayor robustez y despliegue a la política juvenil nacional, sino que más bien fue el modo como institucionalmente se resolvió una disputa política entre dos agrupaciones kirchneristas: Kolina y La Cámpora.

Una situación que establece claramente una diferencia respecto al periodo anterior es que el propio Ministerio de Desarrollo Social (MDS) manifiesta que el Estado no alberga militantes y agrupaciones, sino que impulsa un «proyecto militante».

El período que se nos presenta actualmente requiere de la implementación de un modelo de gestión integral que vincule la gestión militante con la política, sin la posibilidad de pensar una sin la otra. [...] Para ello se debe tener en cuenta que las y los jóvenes son diferentes a los de una década atrás y necesitan que desde el Estado se diseñen herramientas acordes a los cambios de época que estén transitando. (Ministerio de Desarrollo Social de la Nación \& Unesco, 2011, citado por Vázquez, 2014, p. 95)

En definitiva, desde el 2007 a la fecha estaríamos asistiendo a la consagración de la juventud como «causa militante» y del trabajo en el Estado como «gestión militante». ${ }^{15}$

El análisis de las acciones que la Dinaju/SSJ ha implementado desde el 2006 revela que prioritariamente se dirigen a promover la participación juvenil y son un correlato directo de las interpretaciones y objetivos de esta nueva etapa, ${ }^{16}$ por lo que se diferencian de lo ocurrido durante el periodo neoliberal. ${ }^{17}$

15 Para profundizar sobre estas conceptualizaciones véanse los textos ya referenciados de Melina Vázquez.

16 Del conjunto de políticas públicas de juventud implementadas entre los años 2010 y 2014, el $25 \%$ del total contempla entre sus objetivos promover la participación juvenil y dependen principalmente de los organismos del MDS de la Nación (Vázquez, 2015b).

${ }_{17}$ Durante el kirchnerismo también se desplegó una poderosa batería de políticas sectoriales dirigidas directa o indirectamente a las juventudes que representaron un salto cualitativo en cuanto a la asignación o restitución de derechos respecto a lo realizado en la década de los noventa. Nos referimos a programas como los de terminación de los estudios, los de salud sexual y reproductiva «Conectar igualdad», «Asignación universal por hijo», «Jóvenes con más y mejor trabajo» y las becas Progresar. 
En este sentido, el MDS establece como eje central de las políticas del organismo juvenil el «enfoque promocional y participativo ${ }^{18} \mathrm{e}$ impulsa con estas una mirada y un tratamiento integral de la cuestión juvenil. En 2010, con el cambio de autoridades, se incorpora además la construcción y profundización de vínculos con actores externos al organismo - como sectores académicos y movimientos sociales y políticos-, que terminan por consolidar, al menos discursivamente, la consagración estatal de las y los jóvenes como sujetos de derechos, y no como simples objetos o destinatarios de sus políticas, y la necesidad de diseñar e implementar políticas integrales de juventud (Vázquez, 2015a).

A pesar de las nuevas líneas de acción y los enfoques de abordaje que el posneoliberalismo kirchnerista propició en el OGJ, el análisis de la actuación de este en sus distintos periodos nos permite señalar una serie de continuidades respecto a la década de los noventa, en cuanto a la institucionalidad juvenil específica:

- La Dinaju-SSJ tuvo escasa sistematicidad y organicidad en su institucionalidad, así como una insuficiente influencia y articulación a la hora de diseñar e implementar las políticas sectoriales sobre juventud del gobierno. Además, el organismo vio limitada su capacidad de incidir en la consolidación de miradas integrales y generacionales en la estructura orgánica nacional y en la implementación del conjunto de políticas enfocadas en las juventudes. En relación con el punto anterior, se detectó en las dependencias del Estado nacional competentes en la materia, la convivencia de distintos paradigmas y conceptualizaciones sobre la cuestión juvenil, a veces incluso contradictorios y antagónicos. ${ }^{19}$

- El OGJ, en su rol de articulador y promotor del abordaje de la cuestión juvenil en el ámbito federal, tuvo serias dificultades para cumplir sus funciones y tareas institucionales como interlocutor entre el Estado nacional y los organismos subnacionales. En este sentido, la orientación y afinidad política de los gobiernos provinciales y locales con el nacional funcionaron, igual que en el periodo neoliberal, como puerta o barrera a la implementación de sus políticas y programas sobre juventud en los territorios provinciales.

18 Dicho enfoque consiste en «propiciar políticas de promoción y orientadas al desarrollo y/o construcción de ciudadanía, de manera que se efectivice la participación de los/las jóvenes en el diseño de su realidad a partir de la convocatoria efectiva de plantear y resolver sus problemáticas («Políticas sociales de juventud: aprendizajes y desafíos», Ministerio de Desarrollo Social de la Nación, 2007)» (Núñez et al., 2015, p. 110).

19 El análisis comparativo de Núñez et al. (2015) de los paradigmas del dispositivo «Organizarnos para transformar», de la SSJ, y el programa «Jóvenes con más y mejor trabajo», del Ministerio de Trabajo, Empleo y Seguridad Social, ilustra claramente las contradicciones divisadas. 
- En el diseño, implementación y monitoreo de los programas de la Dinaju se reconoce un continuidad del carácter focalista y cortoplacista. Asimismo, los OGJ dispusieron de exiguos presupuestos durante el periodo.

- En general, durante el kirchnerismo el abordaje estatal de la cuestión juvenil y las principales políticas enfocadas en el tema, en términos de universalidad de las prestaciones, gran cantidad de beneficiarios, elevados montos de inversión presupuestal y mayor desarrollo de institucionalidad, se pueden atribuir a entidades sectoriales diferentes del OGJ. Esta particularidad se corresponde con lo sucedido en el periodo neoliberal.

En definitiva, en el periodo posneoliberal de gobierno, de fuerte tendencia estadocéntrica, la institucionalidad juvenil específica nacional continuó afectada por escaso desarrollo y consolidación, mostrando significativas coincidencias con la débil actuación del OGJ en el periodo neoliberal. En cambio, pareciera que en lo referido a los enfoques sobre la juventud, paradigmas de los programas y modelos de abordaje de las políticas en dicho organismo, las diferencias se tornaron más evidentes.

Si bien los trabajos citados en este apartado no tratan de establecer una correlación causal entre los proyectos de gobierno y el desarrollo de institucionalidad de los OGJ, del conjunto de continuidades observadas en las distintas comparaciones se desprende que el vínculo de las definiciones político-ideológicas (en la categoría proyecto de gobierno) con un tipo particular de institucionalidad juvenil de un ámbito específico no es tan claro o definido.

A partir de este hallazgo, resulta necesario avanzar sobre otros posibles factores causales que operarían en la similar configuración de las actuaciones de los OGJ entre 1989 y 2015, y si bien no es algo que esté al alcance del presente artículo, constituyen puntos que se desarrollarán en futuras líneas investigativas para profundizar sobre lo expuesto en esta primera exploración de revisión del tema (de corte descriptivo), con estudios que incluirían esquemas explicativos, para dar mayor robustez a los indispensables estudios de desarrollo de institucionalidad juvenil.

\section{Reflexiones finales}

Tras la revisión y el análisis de estudios y bibliografía especializada, podemos afirmar que existen múltiples rupturas y continuidades, entre el periodo neoliberal y el 
posneoliberal, en la forma de abordar la cuestión juvenil. En términos generales, entre los años 2003 y 2015 se observa una clara potencia y fortaleza de los gobiernos posneoliberales en el despliegue de importantes políticas juveniles sectoriales, con criterio universalizador, que van más allá de las preferencias focalizadas del menemismo. Durante el kirchnerismo hubo una apuesta por la restitución de derechos y por mejorar las condiciones de vida de la población, evidenciada en indicadores de cómo se trabajó la cuestión social con una nueva visión, como la pobreza, la indigencia y la distribución del ingreso, por nombrar solo algunos aspectos. De acuerdo con la bibliografía revisada, este tipo de políticas se encontraría en directa consonancia con un proyecto de gobierno posneoliberal que promovió un nuevo modelo de acumulación y de matriz estadocéntrica.

Puntualizando en el vínculo entre proyecto de gobierno y características de los OGJ, entendidos como evidentes señales de rupturas respecto al periodo neoliberal, en la etapa 2003-2015 se encontró una notoria diferencia en las definiciones y paradigmas sobre la cuestión juvenil y en los sentidos de la intervención del OGJ, propios de un proyecto posneoliberal que se inclina hacia políticas de participación, empoderamiento y desarrollo de autonomía juvenil. Como contrapartida, el periodo neoliberal (1989-2003) se caracterizó por políticas juveniles compensatorias, de control social de riesgos y orientadas a la preparación de las y los jóvenes para su ingreso al mercado laboral y al mundo adulto.

Sin embargo, en cuanto a las continuidades, pareciera que el escaso desarrollo de capacidades estatales de los OGJ es una característica presente en ambos proyectos. Es decir, entre 1989 y 2003, con la profundización del modelo neoliberal, y hasta la consolidación de un proyecto posneoliberal en el periodo 2003-2015, el organismo nacional juvenil específico conservó ciertas «inmanencias»: bajo presupuesto para sacar adelante sus gestiones, poca capacidad articuladora y de influencia para implementar políticas juveniles sectoriales y transversales con perspectiva generacional, miradas y programas cortoplacistas y escaso poder político del organismo que funciona como trampolín central de las trayectorias políticas de sus funcionarios. Lo anterior desarticula y anula la potencia del desempeño de los OGJ en el diverso y amplio entramado institucional que los gobiernos nacionales desplegaron para abordar la cuestión juvenil. En definitiva, aparece como un organismo con insuficiente desarrollo de capacidades estatales.

De lo desarrollado surge como interrogante, particularmente enfocado en los proyectos posneoliberales en Argentina (tras el cambio de paradigmas, el aumento de inversión, la restitución y promoción de amplios derechos en la ciudadanía —entre ellos, de las juventudes-), el porqué no se traduce todo esto en cambios en los organismos guber- 
namentales juveniles y en su actuación. De acuerdo con la teoría, e incluso el propio sentido común, se tendería a suponer que ante semejantes cambios en la forma de abordar la nueva cuestión social debieran necesitarse nuevas reglas, modos de organizarse y, principalmente, una nueva estructura orgánica para desplegar institucionalidad y políticas enfocadas, en sentido estricto, en la juventud.

A partir de los hallazgos expuestos, cabe afirmar que en ninguno de los proyectos de gobierno analizados ha habido un importante desarrollo en materia de institucionalidad juvenil, particularmente de los OGJ.

A modo de cierre, cabría pensar que en el caso del kirchnerismo se ha producido una particular tensión y contradicción entre el plano discursivo sobre lo que se entendía como Estado nacional respecto a la forma de abordar la cuestión juvenil y cómo se traducía en términos de despliegue institucional en el caso concreto de los OGJ y sus políticas:

1) Si bien los gobiernos kirchneristas, de manera novedosa (si se compara con gobiernos anteriores), hicieron hincapié en la categoría joven y en la cuestión juvenil a la hora de introducirlas en la agenda pública, el diseño institucional que dieron a los organismos específicos de juventud para abordar esos temas fue similar al de los organismos creados durante las décadas de los ochenta y noventa. Además, en la práctica político-partidaria de los gobiernos, los OGJ funcionaron más como órganos de negociación y premiación política, para distintos sectores o actores de la coalición de gobierno.

2) Mientras que los discursos de funcionarios de los OGJ y las políticas diseñadas por el organismo adoptaban nuevos paradigmas sobre las juventudes y proponían enfoques generacionales, los OGJ continuaban dependiendo del Ministerio de Desarrollo Social, no tenían injerencia en las políticas sectoriales, ni en la consolidación de una perspectiva generacional ni en la gestión. El presupuesto de los OGJ se mantuvo en bajos montos y representó una pequeña proporción del presupuesto del MDS y del presupuesto nacional, no muy diferente del que se les asignó en la década anterior.

3) Mientras que en la política juvenil sectorial se decidió avanzar con importantes programas, concebidos con criterios de universalidad (en contraposición a la focalización neoliberal), el OGJ diseñó e implementó pequeños programas focalizados, semejantes a los de un OGJ subnacional.

Son muchos los países latinoamericanos que enfrentan déficits de desarrollo y baja calidad institucional de los OGJ nacionales (Rodríguez, 2003). De acuerdo con este 
autor, comparten como problema central la sistemática incapacidad de impulsar e imponer una perspectiva generacional a sus políticas e instituciones públicas. Entonces, continúa abierto el debate dirigido a definir y desarrollar un tipo de institucionalidad que sea acorde con la generación de bienestar para las juventudes latinoamericanas y caribeñas. Retomando la cita inicial de Dávila-León y Silva, no se pone en duda cuán imprescindible es el desarrollo de institucionalidad para hacer sostenibles las políticas, pero sí cuán necesaria es la institucionalidad específica tal como se viene desplegando. Tal parece que conviene dotar de un enfoque generacional ${ }^{20}$ a las políticas sectoriales relacionadas con la juventud y abandonar los tradicionales OGJ.

La incorporación de este paradigma abriría el camino para encontrar integralidad, transversalidad, continuidad, estabilidad político-institucional y correlación entre el discurso y la práctica en materia de tratamiento de la cuestión juvenil, con lo que se lograrían superar las tensiones y paradojas observadas a lo largo del análisis. En consecuencia, empíricamente se está demostrando que el Estado nacional argentino no ha podido incorporar aún un enfoque generacional para su institucionalidad tanto en su momento neoliberal como - sobre todo y como se esperaría - en su etapa posneoliberal.

Como caracterización general, pensamos que aún no se ha logrado cristalizar dicho enfoque, no solo en el plano de los OGJ, sino en el conjunto de la estatalidad nacional, y que la implementación del mismo constituiría un salto cualitativamente positivo e innovador en la producción estatal relacionada con las juventudes. No obstante, antes de avanzar por ese camino habría que desentrañar, en consonancia con nuestro análisis inicial, si la presuposición de un enfoque generacional depende o no de la variable ideológica proyecto de gobierno.

En definitiva, ¿adoptar un enfoque generacional es indistintamente compatible, esperable y deseable, tanto con los proyectos neoliberales como con los posneoliberales? Nuestra respuesta inicial es que no habría compatibilidad. Desde nuestro enfoque, las instituciones, y más aún las que intervienen en la generación de bienestar, no son neutrales (Roca et al. 2012; Cecchini et al., 2015). En este sentido, la variable ideológica continúa siendo relevante en el momento de explicar las narrativas y paradigmas relacionados con las juventudes. El enfoque neoliberal las homogeneiza según su definición como

2o Según Rodríguez (2018), la perspectiva generacional «permite la construcción de identidades particulares en el marco de un enfoque relacional (con el mundo adulto) y que al mismo tiempo permite impulsar acciones asumiendo la existencia de relaciones de poder (en este caso, en mano de adultos)» (p. 35). 
grupo de riesgo o etapa del ciclo vital, mientras que los proyectos posneoliberales, en contrapartida, definen a las y los jóvenes como sujetos de derecho (Rodríguez, 2018).

A partir de las características detectadas en los OGJ que operaron entre 1989 y 2015 surge la duda de si el marco político-ideológico de un proyecto de gobierno es la única variable que condiciona y estructura la institucionalidad juvenil específica, o si hay que buscar respuestas en otro tipo de interpretaciones.

En este sentido, se propone ampliar el esquema de variables para comprender nuestro objeto de estudio. Una posible tentativa, desde una posición neoinstitucionalista, es concebir las instituciones como parte de una matriz política y reconocer que estas influyen sobre las variables ideológicas y socioeconómicas y también sobre los actores; pero aceptar que, a la vez, estas últimas variables influyen sobre las instituciones (Acuña, 2013). Es decir que, además de la variable ideológica (proyecto de gobierno) y del contexto socioeconómico sobre el cual se despliega un modelo político, se propone incorporar al esquema analítico las injerencias de los actores y el marco político-institucional (y las interacciones entre estas variables) como potenciales factores explicativos que permitan abrir la «caja negra» de la institucionalidad que produce el Estado sobre la cuestión juvenil.

Esperamos, en un pronto y futuro trabajo, profundizar sobre este tipo de estudios, y ampliar e incluir nuevas variables al esquema analítico, para lograr una mejor comprensión del fenómeno.

\section{Referencias}

Acuña, C. (2013). Cuánto importan las instituciones: gobierno, Estado y actores en la política argentina. Siglo XXI.

Aguilar-Villanueva, L. (1993). El estudio de las políticas públicas. Miguel Ángel Porrúa.

Alegre, P. (2008). Los giros a la izquierda en el Cono Sur: gobiernos progresistas y alternativas de desarrollo en perspectiva comparada. Informe final del concurso «Gobiernos progresistas en la era neoliberal: estructuras de poder y concepciones sobre el desarrollo en América Latina y el Caribe». Consejo Latinoamericano de Ciencias Sociales. Ander-Egg, E. (1995). Introducción a la planificación estratégica. Lumen.

Balardini, S. (1999). Políticas de juventud: conceptos y la experiencia argentina. Última Década, (10), 1-17. 
Balardini, S., \& Hermo, J. (1995). Políticas de juventud en América Latina: evaluación y diseño. Informe Argentina. Facultad Latinoamericana de Ciencias Sociales.

Beretta, D., Galano, N., \& Laredo, F. (2018). Cartografía de políticas públicas de juventudes: reflexiones a partir de sus configuraciones en Rosario. Grupo Editor Universitario.

Bobbio, N. (1995). Izquierda y derecha: razones y significados de una distinción política. Taurus. Bonvillani, A., Palermo, A., Vázquez, M., \& Vommaro, P. (2008). Juventud y política en Argentina (1968-2008): hacia la construcción de un estado del arte. Revista Argentina de Sociología, 6(11), 44-73.

Borzese, D., Botinelli, L., \& Luro, V. (2009). Hacia una ley nacional de juventudes en Argentina: análisis de experiencias de construcción de leyes de juventud en países de América Latina. Fundación Carolina; Fundación SES.

Bresser-Pereira, L. C. (2007). El nuevo desarrollismo y la ortodoxia convencional. Economía UNAM, 4(10), 7-29.

Castañeda, J. G. (2006). Latin America's left turn. Foreign Affairs, 85(3), 28-43.

Cecchini, S., Filgueira, F., Martínez, R., \& Rossel, C. (2015). Instrumentos de protección social: caminos latinoamericanos hacia la universalización. Comisión Económica para América Latina y el Caribe.

Comisión Económica para América Latina y el Caribe \& Organización Iberoamericana de la Juventud. (2000). Adolescencia y juventud en América Latina y el Caribe: problemas, oportunidades y desafíos en el comienzo de un nuevo siglo. Celade; OIJ.

Dávila-León, O., \& Silva, J. C. (1999). Políticas de juventud y su expresión en lo local. Última Década (11), 1-31.

Fleury, S. (2000). Reforma del Estado: diseño y gerencia de políticas y programas sociales. Banco Interamericano de Desarrollo; Instituto Interamericano para el Desarrollo Social.

García, A., \& Liguori, M. (2015). Participación política de jóvenes y políticas públicas en la década del ochenta en Argentina: el caso de la creación de la Subsecretaría Nacional de Juventud. Saap.

Giordano, V. (2014). ¿Qué hay de nuevo en las «nuevas derechas»? Nueva Sociedad, (254), 46-56. Golbert, L. (2010). De la sociedad de beneficencia a los derechos sociales. Ministerio de Trabajo, Empleo y Seguridad Social [Argentina].

Krauskopf, D. (2008). Avances y desafíos en la institucionalidad pública en juventud. Revista Observatorio de Juventud, 5(17), 13-21.

Laguado, A., \& Cao, H. (2015). Una administración pública para las sociedades posneoliberales en América Latina (2003-2013). Revista Estado y Políticas Públicas (4), 60-68. 
Lievesley, G. (2009). Is Latin America moving leftwards? Problems and prospects. En G. Liesvesley \& S. Ludlam (eds.), Reclaiming Latin America: Experiments in radical social democracy (153-17o). Zed Books.

Margulis, M. (2001). Juventud: una aproximación conceptual. En Donas Burak (Comp.) Adolescencia y Juventud en América Latina. Libro Universitario Regional (Eulac-GTZ).

Ministerio de Desarrollo Social de la Nación. (2007). Políticas sociales de juventud: aprendizajes y desafíos.

Nazareno, M. (2009). Hacer la diferencia: nueva izquierda, coaliciones y política de la política económica. La Argentina en el contexto latinoamericano, 2003-2007 [Tesis doctoral no publicada]. Facultad Latinoamericana de Ciencias Sociales.

Núñez, P., Vázquez, M., \& Vommaro, P. (2015). Entre la inclusión y la participación: una revisión de las políticas públicas de juventud en la Argentina actual. Juventudes latinoamericanas: prácticas socioculturales, políticas y políticas públicas. Consejo Latinoamericano de Ciencias Sociales.

Osborne, D., \& Gaebler, T. (1992). Reinventing government: How the entrepreneurial spirit is transforming government. Addison Wesley.

Oszlak, O. (1990). La reforma del Estado en la Argentina. Cedes.

Oszlak, O. (1999). De menor a mejor: el desafío de la segunda reforma del Estado. Nueva Sociedad (160), 81-100.

Oszlak, O. (2004). Privatización y capacidad de regulación estatal: una aproximación teóricometodológica. Fondo de Cultura Económica.

Ramírez-Gallegos, F. (2012). Crisis neoliberal y reconfiguraciones estatales: Ecuador y la heterodoxia sudamericana, Revista Linea Sur, 1(2), 84-104.

Repetto, F., \& Masetto, F. (2011). Protección social en la Argentina. Comisión Económica para América Latina y el Caribe.

Rey, M. (2014). Capacidad estatal y poder del Estado en Latinoamérica del siglo XXI: una perspectiva política para el análisis de las políticas públicas y la estatalidad. Revista Estado y Políticas Públicas, (2), 115-139.

Roca, E., Golbert, L., \& Lanari, M. E. (2012). ¿Piso o sistema integrado de protección social? Una mirada desde la experiencia argentina. Ministerio de Trabajo, Empleo y Seguridad Social [Argentina].

Rodríguez, E. (2000). Juventud y políticas públicas en América Latina: experiencias y desafíos desde la gestión institucional. Última Década, 8(13), 35-58. http://dx.doi.org/ 10.4067/So718-22362000000200003 
Rodríguez, E. (2003). Políticas públicas de juventud en América Latina: de la construcción de espacios específicos al desarrollo de una perspectiva generacional. Revista Latinoamericana de Ciencias Sociales, Niñez y Juventud, 1(2), 15-43.

Rodríguez, E. (2015a). ¿Existe alguna correlación entre tipos de gobierno y desarrollo de políticas públicas de juventud en América Latina? Notas iniciales para una construcción colectiva de respuestas pertinentes. Centro Latinoamericano sobre Juventud; Unesco.

Rodríguez, E. (2015b). Crecimiento económico, desarrollo humano y políticas públicas de juventud en América Latina: sintonías y disonancias. Centro Latinoamericano sobre Juventud; Unesco.

Rodríguez, E. (2018). Emputad@s y disputad@: miradas neoliberales, neoconservadoras y neodesarrollistas sobre l@s jóvenes en América Latina. Consejo Latinoamericano de Ciencias Sociales. https://doi.org/10.2307/j.ctvfjd17k.4

Rovira-Kaltwasser, C. (2011). Toward post-neoliberalism in Latin America? Latin American Research Review, 46(2), 225-234. https://doi.org/10.1353/lar.2011.0029

Sader, E. (2008). Posneoliberalismo en América Latina. Consejo Latinoamericano de Ciencias Sociales.

Stoessel, S. (2014). Giro a la izquierda en la América Latina del siglo XXI: revisitando los debates académicos. Polis, 13(39), 123-149. http://dx.doi.org/10.4067/ So718-65682014000300007

Stokes, S. (2008). Globalization and the Left in Latin America. Yale University.

Tirenni, J. A. (2013). La política social argentina ante los desafíos de un Estado inclusivo, 2003-2013. Estado y Políticas Públicas, 1(1) 123-140.

Tokatlian, J. G. (2009, 13 de julio). Neogolpismo. Página/12. https://www.pagina12.com.ar/ diario/elmundo/subnotas/128159-41146-2009-07-13.html

Vázquez, M. (2014). «Militar la gestión»: una aproximación a las relaciones entre activismo y trabajo en el Estado a partir de las gestiones de gobierno de Cristina Fernández de Kirchner en Argentina. Apuntes. Revista de Ciencias Sociales, 41(74), 71-102. https://doi.org/10.21678/apuntes.74.703

Vázquez, M. (2015a). Entre la movilización y el Estado: las políticas participativas de juventud en la argentina actual. Última Década, 23(43), 163-206. http://dx.doi.org/ $10.4067 /$ So718-22362015000200007

Vázquez, M. (2015b). Del que se vayan todos a militar por, para y desde el Estado: desplazamientos y reconfiguraciones del activismo juvenil y las causas militantes luego de la crisis del año 2001 en Argentina. En J. M. Valenzuela (Comp.), El sistema 
es antinosotros: culturas, movimientos y resistencias juveniles. Gedisa; Colef; Universidad Autónoma Metropolitana.

Viguera, A. (2000). Estado, empresarios y reformas económicas: en busca de una perspectiva analítica integradora. Zona abierta, (90-91), 161-203.

Vilas, C. (2012). Instituciones: ni tanto ni tampoco. Revista Aportes, 18(30), 43-52. 